\title{
Tests of the SIBYLL 2.3 high-energy hadronic interaction model using the KASCADE-Grande muon data
}

J.C. Arteaga-Velázquez ${ }^{1, \star}$, D. Rivera-Rangel ${ }^{1, \star \star}$, W.D. Apel ${ }^{2}$, K. Bekk ${ }^{2}$, M. Bertaina ${ }^{3}$, J. Blümer $^{2,4,14}$, H. Bozdog ${ }^{2}$, I.M. Brancus ${ }^{5}$, E. Cantoni ${ }^{3,6,15}$, A. Chiavassa ${ }^{3}$, F. Cossavella ${ }^{4,16}, K$. Daumiller $^{2}$, V. de Souza ${ }^{7}$, F. Di Pierro ${ }^{3}$, P. Doll ${ }^{2}$, R. Engel ${ }^{2}$, D. Fuhrmann ${ }^{8,17}$, A. Gherghel-Lascu ${ }^{5}$, H.J. Gils ${ }^{2}$, R. Glasstetter ${ }^{8}, C$. Grupen ${ }^{9}, A$. Haungs $^{2,18, \star \star \star}, D$. Heck ${ }^{2}, J . R$. Hörandel ${ }^{10}, T$. Huege ${ }^{2}$, K.H. Kampert ${ }^{8}$, D. Kang ${ }^{2}$, H.O. Klages ${ }^{2}$, K. Link ${ }^{4}$, P. Łuczak ${ }^{11}$, H.J. Mathes ${ }^{2}$, H.J. Mayer ${ }^{2}$, J. Milke $^{2}$, B. Mitrica ${ }^{5}$, C. Morello ${ }^{6}$, J. Oehlschläger ${ }^{2}, S$. Ostapchenko ${ }^{12}$, T. Pierog ${ }^{2}, H$. Rebel ${ }^{2}, M$.

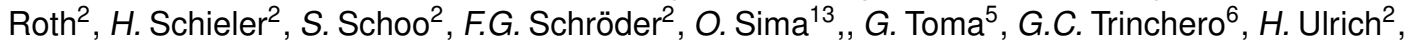
A. Weindl ${ }^{2}$, J. Wochele ${ }^{2}$, and J. Zabierowski ${ }^{11}$

${ }^{1}$ Institute of Physics and Mathematics, Universidad Michoacana de San Nicolás de Hidalgo, Morelia, Mexico

${ }^{2}$ Institut für Kernphysik, KIT - Karlsruher Institut für Technologie, Germany

${ }^{3}$ Departimento di Fisica, Università degli Studi di Torino, Italy

${ }^{4}$ Institut für Experimentelle Teilchenphysik, KIT - Karlsruher Institut für Technologie, Germany

${ }^{5}$ Horia Hulubei National Institute of Physics and Nuclear Engineering, Bucharest, Romania

${ }^{6}$ Osservatorio Astrofisico di Torino, INAF Torino, Italy

${ }^{7}$ Universidade de São Paulo, Instituto de Física de São Carlos, Brasil

${ }^{8}$ Fachbereich Physik, Universität Wuppertal, Germany

${ }^{9}$ Department of Physics, Siegen University, Germany

${ }^{10}$ Department of Astrophysics, Radboud University Nijmegen, The Netherlands

${ }^{11}$ National Centre for Nuclear Research, Department of Astrophysics, Lodz, Poland

${ }^{12}$ Frankfurt Institute for Advanced Studies (FIAS), Frankfurt am Main, Germany

${ }^{13}$ Department of Physics, University of Bucharest, Bucharest, Romania

${ }^{14}$ Now: Head of Division V at KIT - Karlsruher Institut für Technologie, Germany

${ }^{15}$ Now at: Istituto Nazionale di Ricerca Metrologica, INRIM, Torino, Italy

${ }^{16}$ Now at: DLR Oberpfaffenhofen, Germany

${ }^{17}$ Now at: University of Duisburg-Essen, Duisburg, Germany

${ }^{18}$ Spokesperson KASCADE-Grande

\begin{abstract}
The KASCADE-Grande observatory was a ground-based air shower array dedicated to study the energy and composition of cosmic rays in the energy interval $E=1$ $\mathrm{PeV}-1 \mathrm{EeV}$. The experiment consisted of different detector systems which allowed the simultaneous measurement of distinct components of air showers (EAS), such as the muon content. In this contribution, we study the total muon number and the lateral density distribution of muons in EAS detected by KASCADE-Grande as a function of the zenith angle and the total number of charged particles. The attenuation length of the muon content of EAS is also measured. The results are compared with the predictions of the SIBYLL 2.3 high-energy hadronic interaction model.
\end{abstract}

\footnotetext{
${ }^{\star}$ e-mail: arteaga@ifm.umich.mx

$\star \star$ e-mail: drivera@ifm.umich.mx

$\star \star \star$ e-mail: andreas.haungs@kit.edu
} 


\section{Introduction}

Hadronic interaction models are an important element in the analysis of data from extensive air showers (EAS) induced by cosmic rays in the atmosphere. However, they are subject to theoretical and experimental uncertainties, which may hamper cosmic-ray studies. Recently, a lot of progress has been done towards the reduction of such uncertainties motivated, in part, by the results of the measurements of the LHC [1]. In this regard, one of the models that has been updated is SIBYLL [2].

In this work, muon predictions of the post-LHC model SIBYLL 2.3 will be tested using the data of the KASCADE-Grande observatory [3]. Shower muons are employed for these tests since they are very sensitive to the early hadronic processes occurring in the shower [4]. This way a failure of the model to describe the observed muon data of EAS may imply a problem in the description of the hadronic physics of air showers.

\section{The KASCADE-Grande observatory}

KASCADE-Grande was a ground-based air-shower observatory dedicated to investigate the energy spectrum, the elemental composition and the arrival direction of cosmic rays in the energy range from $10^{15}$ and $10^{18} \mathrm{eV}$ [3]. The instrument was located at the Karlsruhe Institute of Technology, Campus North (110 m a.s.l.) in Karlsruhe, Germany and consisted of several detector systems aimed to measure with high precision different components and properties of the EAS (c.f. fig. 1, left). For instance, the shower size or total number of charged particles (electrons plus muons) $\left(E_{t h}>3 \mathrm{MeV}\right)$, the shower core position at ground and the angle of incidence of the event were estimated from the data collected with the Grande array, which was composed by 37 scintillator detectors separated by a mean distance of $137 \mathrm{~m}$ and distributed over a surface of $0.5 \mathrm{~km}^{2}$. Meanwhile the total number of muons $\left(E_{t h}>230 \mathrm{MeV}\right)$ was obtained from local muon density measurements performed with 192 shielded detectors belonging to a $200 \times 200 \mathrm{~m}^{2}$ array called KASCADE [3]. Systematic uncertainties for $N_{c h}$ and $N_{\mu}$ were estimated as described in [3] and [5], respectively. They were found to be $\leq 15 \%$ and $\leq 25 \%$ for each case.

\section{Experimental data and MC simulations}

To perform the present studies, we have considered measured events collected during the full data acquisition period of the experiment, i.e., from December 2003 up to November 2012. On the other hand, in order to diminish the effect of systematic uncertainties in the results of our analyses, we applied several selection cuts to the data. In particular, we considered only events that were measured during stable data acquisition runs with no hardware problems. In addition, we included events that passed successfully the full reconstruction chain [3]. Besides, the EAS cores were required to be located within the limits of a central area of $2.2 \times 10^{5} \mathrm{~m}^{2}$ inside the KASCADE-Grande array and within radial distances in the interval $R=[100 \mathrm{~m}, 600 \mathrm{~m}]$ measured from the center of the KASCADE array. On the other hand, we rejected events with $\theta \geq 40^{\circ}$. Finally, low energy events, which have poor reconstructed muon numbers, were removed from the data sample by requiring showers that activated more than 11 Grande stations and had a high $N_{\mu}$ number (specifically $\gtrsim 3 \times 10^{4}$ and $\gtrsim 4 \times 10^{4}$ for data with $\theta \lesssim 30^{\circ}$ and $30^{\circ} \lesssim \theta<40^{\circ}$, respectively). Using the above selection cuts, we got an experimental sample with about $1.24 \times 10^{7}$ events and an effective time of $\Delta t=1.6 \times 10^{8} \mathrm{~s}$. 

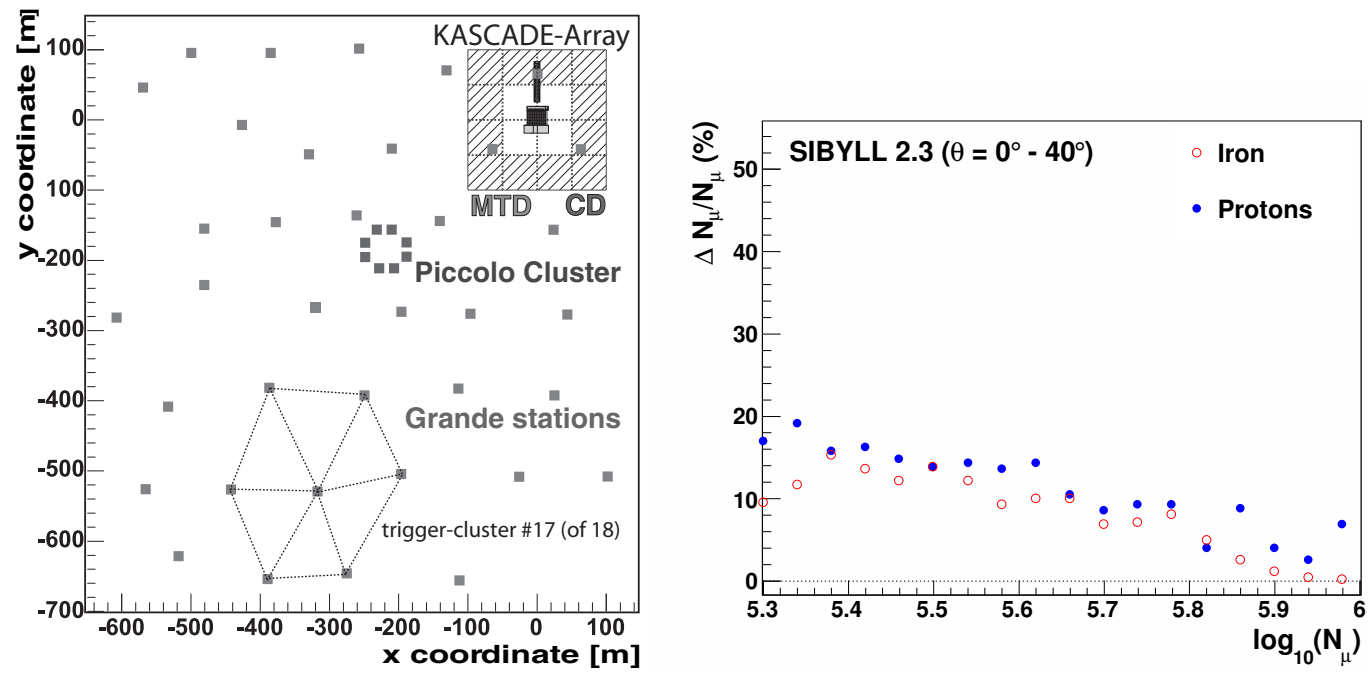

Figure 1. Left: The KASCADE-Grande experiment [5]. Small squares represent the Grande stations. The KASCADE array is seen at the upper right hand of the figure. Close to the center of KASCADE, the Muon tracking detector (MTD) and the Central detector (CD) are shown. KASCADE detectors are arranged in 16 clusters (big squares). The outer 12 clusters contain the 192 shielded plastic scintillator stations employed for the muon measurements. One example of a Grande-trigger is shown. It consists of 6 activated stations in hexagonal configuration plus a central one. Right: Systematic error (reconstructed minus true parameter) of the total number of muons in EAS measured with KASCADE-Grande as a function of the estimated shower muon content. The error was estimated using simulations with SIBYLL 2.3.

On the other hand, the MC simulations were built using SIBYLL 2.3 [2] and Fluka 2011.2 [6] as high- and low-energy $\left(E_{h} \leq 200 \mathrm{GeV}\right)$ hadronic interaction models, respectively. The production and development of the shower were simulated with CORSIKA 7.5 [7], while the response of the detector to the passage of the shower, with a GEANT 3.21 [8] based program. MC events were generated for zenith angles $\theta<42^{\circ}$ and the energy interval from $E=10^{14} \mathrm{eV}$ to $3 \times 10^{18} \mathrm{eV}$ using an $E^{\gamma}$ primary spectrum with spectral index $\gamma=-2$. For the analysis, the MC events were weighted in order to simulate an $E^{-3}$ power-law spectrum, which is in better agreement with the experimental data (see, for example, [9]). MC data samples were produced for five primary nuclei: $\mathrm{H}, \mathrm{He}, \mathrm{C}, \mathrm{Si}$ and $\mathrm{Fe}$, each of them with roughly the same number of events. In total, $\sim 1.28 \times 10^{7} \mathrm{MC}$ showers were generated. All of them were processed with the same reconstruction algorithm employed with the experimental events [3]. In addition, for the analysis we have applied the same selection cuts on the MC sample that were used with the measured data.

According to MC simulations the maximum efficiency is reached for $\log _{10}(E / \mathrm{GeV})=7.2 \pm 0.3$ and $\log _{10}\left(N_{\mu}\right)=5.3 \pm 0.3$ depending of the primary particle and the arrival direction of the EAS. On the other hand, the mean shower core and angular resolutions were found to be $\leq 11 \mathrm{~m}$ and $\leq 0.7^{\circ}$, respectively, for our selected data sample. Finally, the bias in the muon size resulted to be smaller than $20 \%$ for $\log _{10}\left(N_{\mu}\right)>5.3$ (see fig. 1, right). We have improved the accuracy of $N_{\mu}$, for both $\mathrm{MC}$ and measured data, by correcting the reconstructed muon number for systematic biases using the correction function defined in [9]. This way, the final bias on $N_{\mu}$ becomes smaller than $8 \%$. 


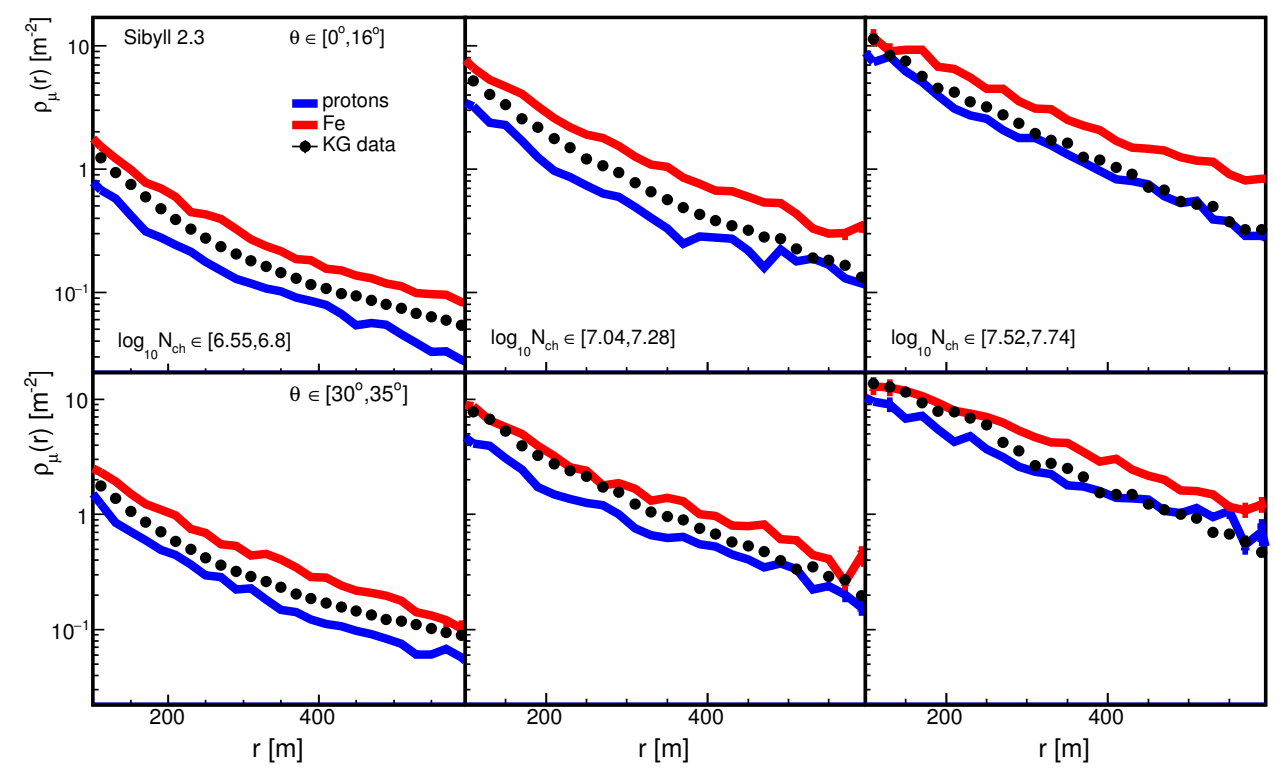

Figure 2. Mean muon lateral density distributions of EAS measured with KASCADE-Grande (points) compared with the predictions of SIBYLL 2.3 for iron (red upper lines) and proton (lower blue lines) primaries [10]. Statistical error bars in measured data are smaller than the size of the markers. From MC simulations the energy scale of the above data spans the interval from $E \sim 10^{7.2} \mathrm{GeV}$ to $10^{9} \mathrm{GeV}$. The primary energy was estimated eventby-event from both $N_{c h}$ and the corrected $N_{\mu}$ using a formula derived from SIBYLL 2.3 simulations (following the procedure described in [9]).

\section{Testing the predictions of SIBYLL 2.3 for shower muons}

\subsection{Muon radial density distributions}

The aim of this analysis is to compare the predictions of SIBYLL 2.3 and the KASCADE-Grande measurements for the mean muon lateral distributions at the shower plane, $\rho_{\mu}(r)$, at different zenith angles and $\log _{10}\left(N_{c h}\right)$ intervals. For this purpose, we divided our data in five bins of $\theta$, each of them with equal acceptance: $\left[0^{\circ}, 16.71^{\circ}\right],\left[16.71^{\circ}, 23.99^{\circ}\right],\left[23.99^{\circ}, 29.86^{\circ}\right],\left[29.86^{\circ}, 35.09^{\circ}\right]$ and $\left[35.09^{\circ}, 40^{\circ}\right]$. Next, we subdivided these data sets in bins of $\log _{10}\left(N_{c h}\right)$ : [6.0,6.55], [6.55, 6.8], [6.8, 7.04], [7.04, 7.28], [7.28, 7.52], [7, 52, 7.74] and [7.74, 8.0]. Then, for each $\theta$ and shower size intervals we built the mean measured $\rho_{\mu}(r)$ distributions (neglecting corrections due to atmospheric absorption when projecting on the shower disk plane). Finally, we compared these experimental distributions against the corresponding predictions from SIBYLL 2.3 for pure protons and iron nuclei, respectively. In fig. 2 we show a plot with results for two zenith angle intervals and three distinct shower size ranges. In particular, we observe that the measured distributions are within the MC predictions. In general, that holds for vertical and inclined showers with $\log _{10}\left(N_{c h}\right)<7.74$. For higher values of the total number of charged particles, we found some deviations, but here statistical fluctuations avoid us to drive a conclusion. 


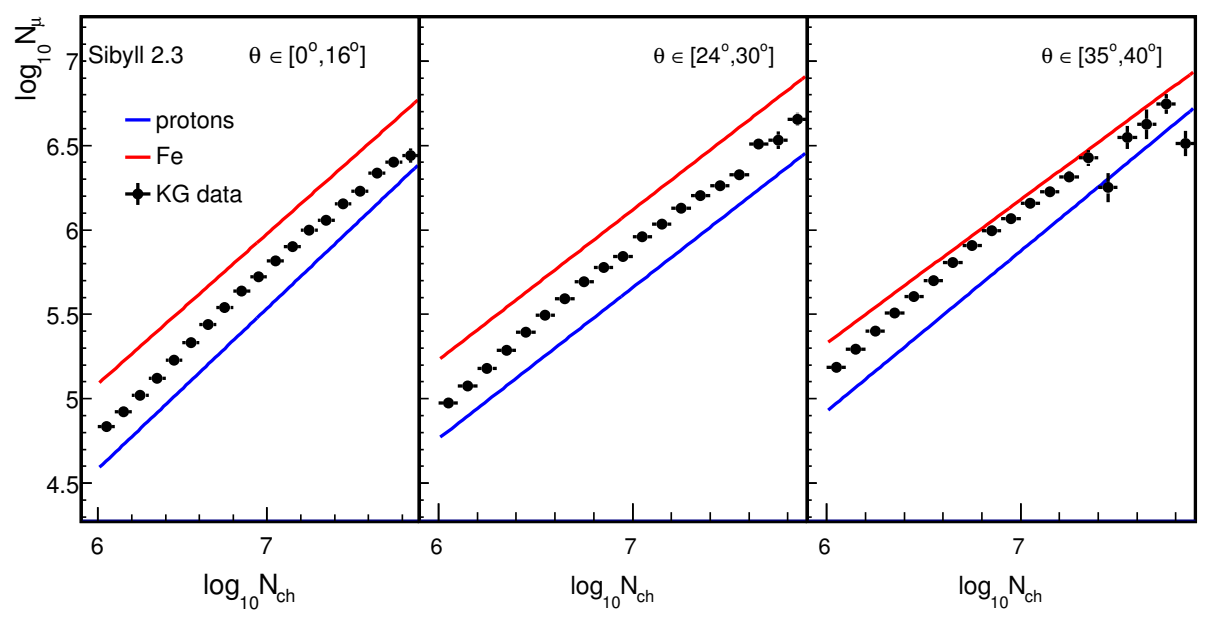

Figure 3. Logarithm of the corrected muon size versus the logarithm of the total number of charged particles in air showers as measured with the KASCADE-Grande detector (black points). Measurements are confronted with predictions of SIBYLL 2.3 for iron nuclei (upper red lines) and protons (lower blue lines) [10]. Vertical error bars represent the errors on the mean. Data is shown for the interval $N_{c h}=10^{6}-10^{7.9}$, which roughly corresponds to mean primary energies between $E \sim 10^{6.8} \mathrm{GeV}$ to $10^{9.1} \mathrm{GeV}$ for the measured data. The energies were estimated event-by-event from both $N_{c h}$ and the corrected $N_{\mu}$ using a formula derived from SIBYLL 2.3 simulations (following the procedure described in [9]).

\subsection{The total muon number}

In this study, we compared the measurements for the corrected muon number as a function of the shower size with the expectations from the SIBYL 2.3 model for hydrogen and iron nuclei for the $\theta$ ranges described in subsection 4.1. The results for three selected zenith angle intervals are presented in fig. 3. In general, we do not observe any deviation of the measured data from the MC simulations. However, we see that for increasing zenith angles the mean composition tends to be heavier, which could be attributed to a mismatch between the predicted and the measured zenith angle evolutions of the shower muon content of EAS. This point was investigated in the analysis of the next subsection.

\subsection{The muon attenuation length}

In order to study the behaviour of the corrected $N_{\mu}$ with $\theta$ for air showers, we calculated the attenuation length of muons in the Earth's atmosphere, $\Lambda_{\mu}$ using the method of [11]. This parameter is especially useful for cross-checking hadronic interaction models. Recent analyses performed in [11] have shown that the $\Lambda_{\mu}$ predictions of the QGSJET-II-2 [12], QGSJET-II-04 [13], EPOS-LHC [14] and SIBYLL 2.1 [15] hadronic interaction models deviate from the measured value at KASCADE-Grande for data in the primary energy interval from $E \sim 10^{16.3} \mathrm{eV}$ to about $10^{17} \mathrm{eV}$ and zenitn angles $<40^{\circ}$. Besides, it was pointed out in [11] that statistical and systematic errors (associated to instrumental effects, reconstruction/analysis methods, EAS fluctuations, etc.) can not explain the observed anomaly. From the results of the last subsection we suspect that also the prediction of SIBYLL 2.3 could deviate from the experimental value. To test this hypothesis we performed an analysis like that described in [11]. 

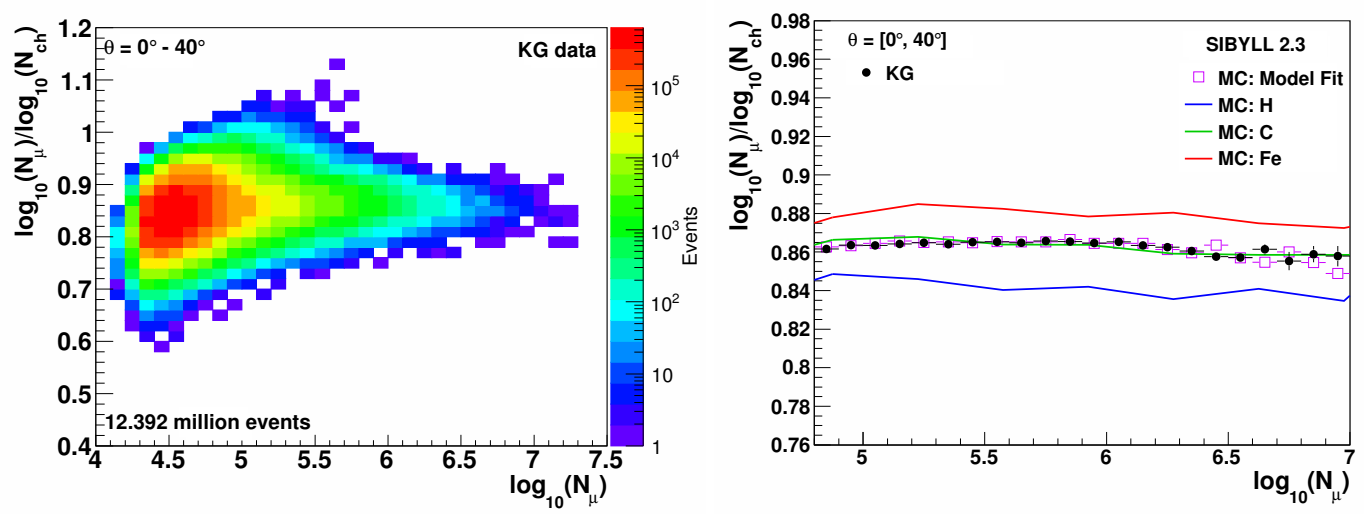

Figure 4. Left: The measured distribution for the ratio $Y=\log _{10}\left(N_{\mu}\right) / \log _{10}\left(N_{c h}\right)$ as a function of the $\log _{10}\left(N_{\mu}\right)$ for selected EAS with $\theta<40^{\circ}$ [10]. The effective observation time of the data sample is $\Delta t=1.6 \times 10^{8} \mathrm{~s}$. Right: The mean of the experimental $Y$ parameter for the data shown on the left against $\log _{10}\left(N_{\mu}\right)$ (black circles). The respective mean is also shown for predictions of SIBYLL 2.3 for protons (lower blue line), carbon (middel green line), iron nuclei (upper red line) and our fitted composition model (open squares, see text). The MC simulations include the full detector simulation and the EAS reconstruction procedures [10].

First, in order to reduce the systematic uncertainties due to the unknown cosmic ray composition in the the value of $\Lambda_{\mu}$ predicted by SIBYLL 2.3, we used a composition model derived from the data itself. The procedure is simple. By using a $\chi^{2}$ fit, we find the weight parameters for the MC energy spectra of four mass groups $(\mathrm{H}, \mathrm{He}, \mathrm{C}$, and $\mathrm{Si}+\mathrm{Fe}$ in a $50 \%$ mixture) that minimize the difference between the measured $Y=\log _{10}\left(N_{\mu}\right) / \log _{10}\left(N_{c h}\right)$ vs $\log _{10}\left(N_{\mu}\right)$ event distribution (c.f. fig. 4, left) and the predicted one, the latter for the linear combination of the four mass groups. Before weighting, the MC energy spectra resemble an $E^{-3}$ power-law function. The fit was performed for $\log _{10}\left(N_{\mu}\right) \geq 4.8$ with the following Chi-square function:

$$
\chi^{2}=\sum_{i, j} \frac{\left[n_{i j}^{\text {exp }}-\sum_{A} n_{i j A}^{M C}\left(\mathbf{p}_{A}\right)\right]^{2}}{\left(\sigma_{i j}^{M C}\right)^{2}},
$$

where $n_{i j}^{\text {exp }}$ is the number of experimental events for the bin $\left(Y_{i}, N_{\mu, j}\right)$ of the respective $Y$ distribution, while $n_{i j A}^{M C}$ is the corresponding number of MC events for the above bin and the mass group $A$. The widths of the $Y$ and $\log _{10}\left(N_{\mu}\right)$ bins are 0.02 and 0.1 , respectively. On the other hand, $\sigma_{i j}^{M C}$ is the statistical error of $n_{i j A}^{M C}$ and $\mathbf{p}_{A}$ is a vector with the parameters that define the weights, $\mathbf{w}_{A}(E)$, of the energy spectrum for $A$. The weights are applied event-by-event on the MC data according to its true energy, $E$. They are parameterized using a double power-law formula

$$
\mathbf{w}_{A}\left(E, \mathbf{p}_{A}\right)=p_{0, A} E^{p_{1, A}}\left[1+\left(\frac{E}{p_{4, A}}\right)^{p_{3, A}}\right]^{\left(p_{2, A}-p_{1, A}\right) / p_{3, A}} .
$$

Here the $p_{k, A}$ are free parameters (i.e. the components of $\mathbf{p}_{A}$ ). The reason of using these expressions is due to the presence of breaks around $E=10^{17} \mathrm{eV}$ in the energy spectra of the light [16] and the heavy [17] components of cosmic rays. With the results of the fit and the SIBYLL 2.3 data, we built our composition model. This is shown in fig. 5. The mean values of $Y\left(N_{\mu}\right)$ for our fitted model and the respective measurements are compared in fig. 4, right. In fig. 5, we observe the heavy knee at 


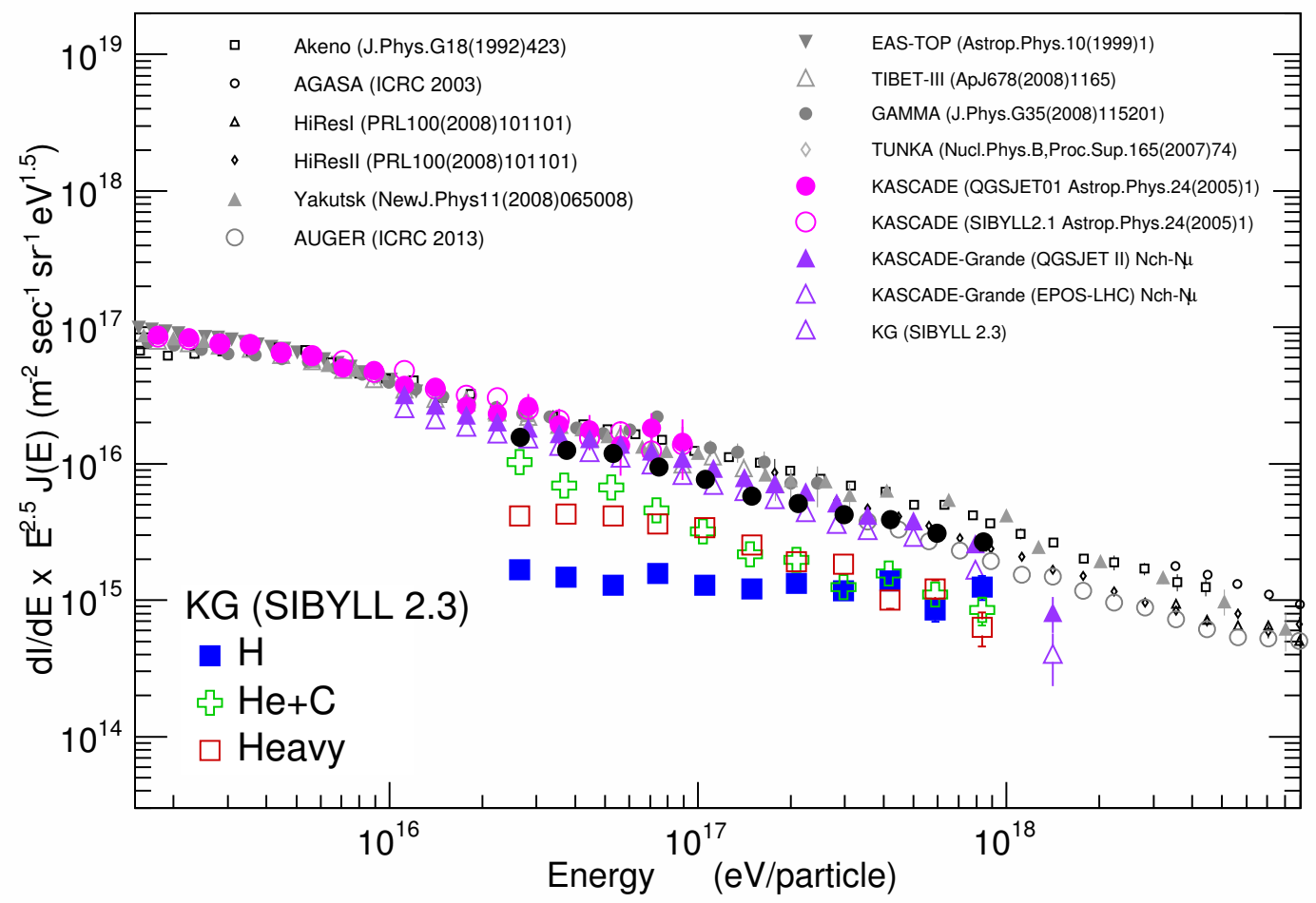

Figure 5. Results of the fit to the measured $Y\left(N_{\mu}\right)$ distributions using SIBYLL 2.3 data and a composition model with four mass groups $(\mathrm{H}, \mathrm{He}, \mathrm{C}$, and $\mathrm{Si}+\mathrm{Fe}$ in a $50 \%$ mixture) each of them following a double power-law energy spectrum [10]. The derived all-particle energy spectrum is shown with black dots. The fitted spectra for protons, $\mathrm{He}+\mathrm{C}$, and the heavy component of cosmic rays are shown in the lower part of the figure with blue full squares, green open crosses and red open squares, respectively. The aforementioned curves are compared with results of other observatories and previous estimations of KASCADE-Grande for the all-particle energy spectrum.

$\log _{10}(E / \mathrm{eV})=16.80 \pm 0.01\left(\Delta \gamma=p_{2}-p_{1}=-0.67 \pm 0.03\right)$. We also see the presence of a proton and a medium $(\mathrm{He}+\mathrm{C})$ component in the data.

Now, following the procedure of [11], we selected a subsample of MC data with radial distances, $R$, between $270 \mathrm{~m}$ and $440 \mathrm{~m}$ and within an area of $8 \times 10^{4} \mathrm{~m}^{2}$ located at the center of the KASCADEGrande array, as they further reduce the systematic bias on $N_{\mu}$. Then, to obtain $\Lambda_{\mu}$, we applied the constant intensity cut (CIC) method that assumes that the intensity of cosmic rays is isotropic in zenith angle [18]. The method consists of selecting $N_{\mu}$ data from different zenith angles by means of cuts applied at fixed frequencies on the integral intensity of $N_{\mu}$ for different zenith angle ranges (see fig. 6 , left). Actually, data selected along these curves should correspond to events with the same primary energy but different shower sizes due to the increasing atmospheric depth at high zenith angles. This dependence is expressed in terms of a typical exponential absorption formula, which is parameterized 

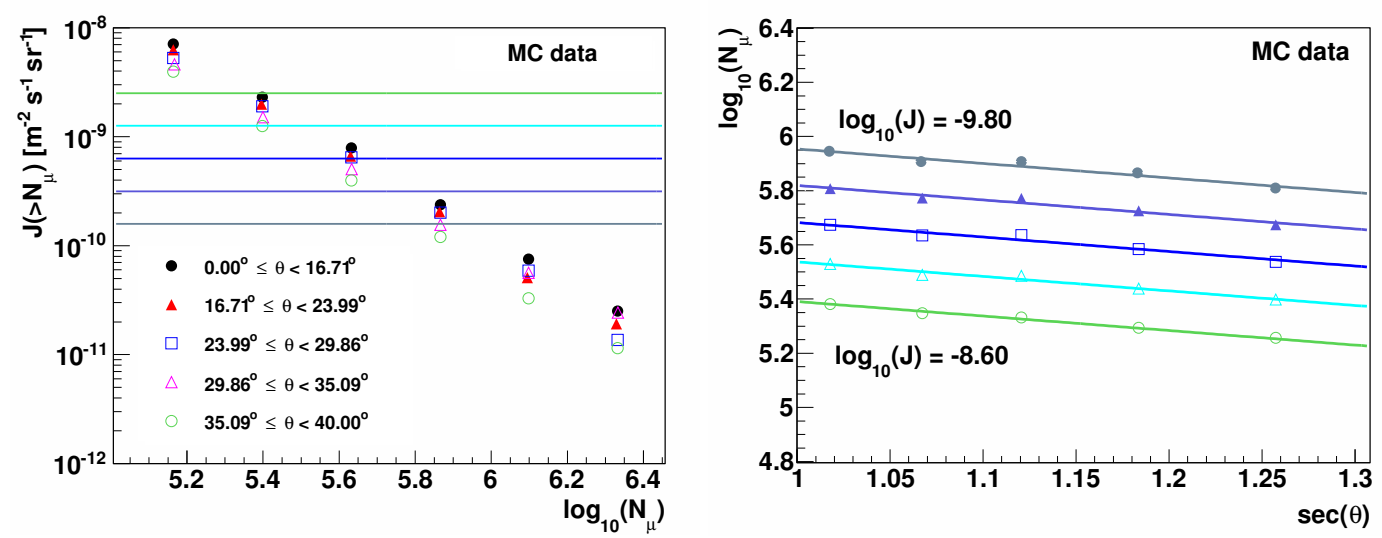

Figure 6. Left: Integral muon intensities derived from a subsample of SIBYLL 2.3 data for five zenith angle intervals using the composition model described in section 4.3. The CIC cuts employed in this work are shown as horizontal lines. Statistical error bars are smaller than the size of the data points. Right: Muon attenuation curves extracted with the CIC method from the MC data. The results of the global fit with equation (3) are shown with solid lines. The cuts are decreasing from bottom to top in units of $\Delta \log _{10}\left[J /\left(\mathrm{m}^{-2} \cdot \mathrm{s}^{-1} \cdot \mathrm{sr}^{-1}\right)\right]=-0.30$. The vertical error bars of the data points are smaller than the size of the symbols and consider statistical uncertainties and errors from interpolation.

as a function of a single $\Lambda_{\mu}[11]$ (see fig. 6 , right):

$$
N_{\mu}(\theta)=N_{\mu}^{\circ} e^{-X_{0} \sec (\theta) / \Lambda_{\mu}},
$$

where $X_{0}=1022 \mathrm{~g} / \mathrm{cm}^{2}$ is the vertical column depth at KASCADE-Grande and $N_{\mu}^{\circ}$, a normalization factor, which depends on the attenuation curve. In our analysis, we divided the MC data into five zenith angles with approximately the same acceptance as in [11] and section 4.1 (see also fig. 6, left) and applied the same CIC cuts described in the abovementioned paper. Using our composition model and the method described, we have calculated $\Lambda_{\mu}$ for SIBYLL 2.3. The result is presented in fig. 7. The systematic error only includes the uncertainty from the CIC method.

In the same plot we show the experimental value obtained in [11] for a subsample of experimental data (collected during the period December 2003 - October 2011) along with its corresponding statistical and total uncertainties. The latter were estimated through detailed studies. The systematic error includes the uncertainty due to the calculation procedure, the uncertainty owing to the muon correction function, the systematic error of the corrected muon number and the corresponding composition and model dependence, the uncertainty owing to the size of the $\theta$ intervals and the uncertainties associated with the shower core position, $R$. MC predictions from QGSJET-II-2, QGSJET-II-04, EPOS-LHC and SIBYLL 2.1 for an $E^{-3}$ model and a mixed composition assumption (all elements on equal abundances) [11] are also shown in our plot. The MC estimations involve, in addition to the ones above, uncertainties due to the spectral index of the primary spectrum, $\gamma$, and due to the primary composition. This last systematic error is estimated by comparing the nominal value, obtained using a mixed composition, with the results for the five primary nuclei employed in simulations. From figure 7 , we observe a hint that the predictions for $\Lambda_{\mu}$ using SIBYLL 2.3 tend to be below the measured data. 


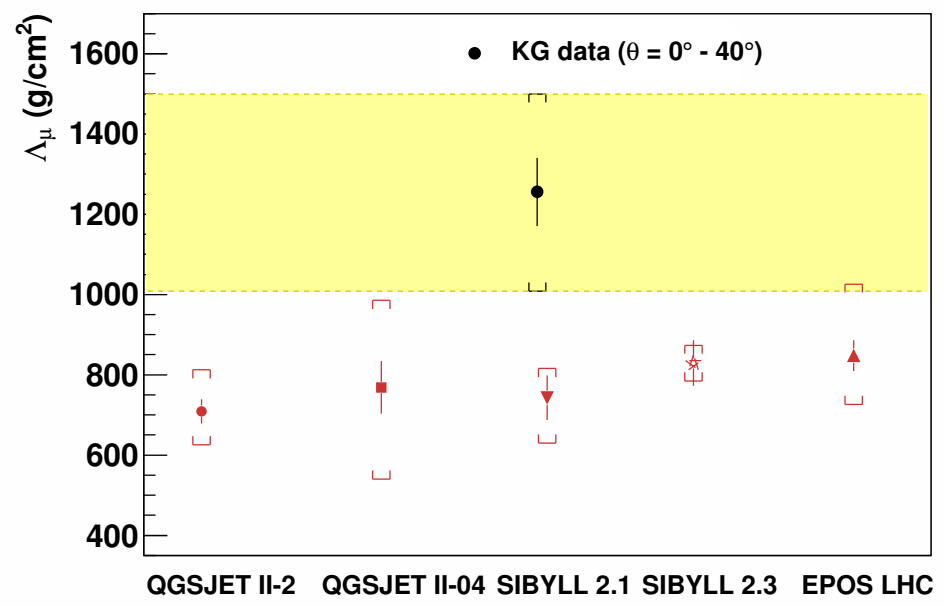

Figure 7. Muon attenuation length measured with the KASCADE-Grande experiment (upper point) compared with predictions of several pre-LHC and post-LHC high-energy hadronic interaction models (lower points) for EAS with core distances within $270 \mathrm{~m}$ and $440 \mathrm{~m}$ from the KASCADE center and with primary energies between $E \sim 10^{16} \mathrm{eV}$ and $\sim 10^{17} \mathrm{eV}$. The total uncertainty (statistical and systematic errors added in quadrature) are shown with squared brackets. Error bars represent statistical uncertainties. The hollowed star is the prediction from SIBYLL 2.3 for our fitted composition model [10] (see text). The experimental result and the predictions from QGSJET-II-02 and 04, EPOS-LHC and SIBYLL 2.1 (for a mixed composition assumption and spectrum $\left.E^{-3}\right)$ are taken from [11].

\section{Conclusions}

We have tested muon predictions of SIBYLL 2.3 against measurements of the KASCADE-Grande observatory for $\rho_{\mu}(r)$ and $N_{\mu}$ at different $\theta$ and $N_{c h}$ ranges, as well as for $\Lambda_{\mu}$. In general, it seems that $\rho_{\mu}(r)$ measurements for $\theta<40^{\circ}$ and $6.0 \leq \log _{10}\left(N_{c h}\right) \leq 7.74$ are within the predictions of the model. For $N_{\mu}$ the agreement is observed in the full shower size range $\left(6.0 \leq \log _{10}\left(N_{c h}\right) \leq 8.0\right)$ and zenith angle intervals $\left(\theta<40^{\circ}\right)$. On the other hand, we see what appears to be a mismatch between the predicted and measured $\Lambda_{\mu}$ values.

\section{Acknowledgments}

The authors would like to thank the members of the engineering and technical staff of the KASCADEGrande Collaboration, who contributed to the success of the experiment. The KASCADE-Grande experiment was supported in Germany by the BMBF and by the 'Helmholtz Alliance for Astroparticle Physics - HAP' funded by the Initiative and Networking Fund of the Helmholtz Association, by the MIUR and INAF of Italy, the Polish Ministry of Science and Higher Education, the Romanian Authority for Scientific Research UEFISCDI (PNII-IDEI grants 271/2011 and 17/2011), and the GermanMexican bilateral collaboration grants (DAAD-CONACYT 2009-2012, 2015-2016). J.C.A.V. acknowledges the partial support of CONACyT (grant CB-2008/106717) and the Coordinación de la Investigación Científica de la Universidad Michoacana. 


\section{References}

[1] S. Ostapchenko, Proc. of the $X X V$ ECRS, eConf C16-09-04.3 (2016), [astro-ph/1612.09461v1].

[2] F. Riehn et al., Proc. of the 34th ICRC, PoS (ICRC2015) 558.

[3] W.D. Apel et al., NIM A 620202 (2010) .

[4] L. Cazon et al., Astrop. Phys. 2172 (2004); L. Cazon et al., Astrop. Phys. 23393 (2005) .

[5] W. D. Apel et al., NIM A 62020 (2010).

[6] A.Fasso et al., Report CERN-2005-10,INFN/TC-05/11, SLAC-R-773 (2005).

[7] D. Heck et al., Report FZKA 6019, Forschungszentrum Karlsruhe (1998).

[8] R. Brun, F. Carminati, CERN Program Library Long Writeup W5013 (1993).

[9] W.D. Apel et al., Astrop. Phys. 36183 (2012).

[10] J.C. Arteaga-Velazquez, D. Rivera-Rangel et al., Proc. of the 35th ICRC, PoS (ICRC2017) 316.

[11] W.D. Apel et al., Astrop. Phys. 9525 (2017).

[12] S.S. Ostapchenko, Nucl. Phys. B (Proc. Suppl.) 151143 (2006); S. Ostapchenko, Phys. Rev. D 74014026 (2006).

[13] S.S. Ostapchenko, Phys. Rev. D 83014018 (2011).

[14] T. Pierog et al., Phys. Rev. C 92034906 (2015).

[15] E.J. Ahn et al., Phys. Rev D 80094003 (2009).

[16] W.D. Apel et al., PRD 87081101 (2013).

[17] W.D. Apel et al., PRL 107171104 (2011).

[18] J. Hersil et al., Phys. Rev. Lett. 622 (1961); D. M. Edge et al., J. Phys. A 61612 (1973). 